Document downloaded from:

http://hdl.handle.net/10251/50794

This paper must be cited as:

Reig, J.; Rubio Arjona, L. (2011). On Simple Estimators of the alpha-mu Fading Distribution. IEEE Transactions on Communications. 59(12):3254-3258. doi:10.1109/TCOMM.2011.080111.090223.

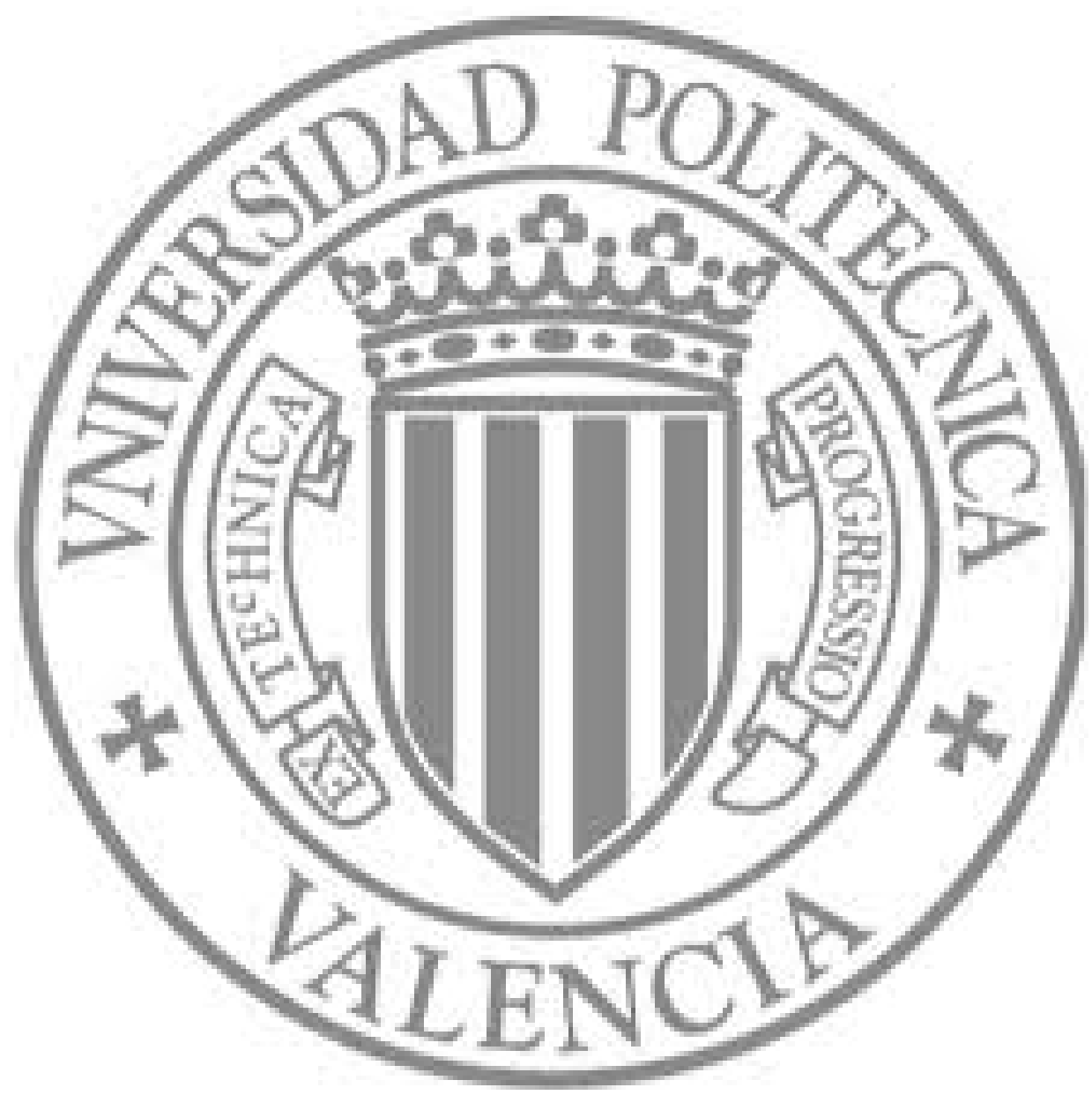

The final publication is available at

http://dx.doi.org/ 10.1109/TCOMM.2011.080111.090223

Copyright Institute of Electrical and Electronics Engineers (IEEE) 


\title{
On Simple Estimators of the $\alpha-\mu$ Fading Distribution
}

\author{
Juan Reig, Member, IEEE, Lorenzo Rubio, Member, IEEE
}

\section{Keywords}

Gamma distribution, fading channels, probability, estimation, land mobile radio propagation factors.

\begin{abstract}
In this letter, new estimators of the $\alpha$ - $\mu$ distribution are derived based on the skewness of the logarithmic $\alpha-\mu$ distribution using the moments method. This distribution has been recently proposed to model the received field strength in nonlinear propagation mediums. Therefore, simple and computationally efficient estimators are required to infer the parameters of the received signal amplitude distribution in nonlinear wireless communication propagation channels. The performance of these new estimators is compared to that obtained with the estimators calculated with the moments method of the $\alpha-\mu$ distribution by solving numerically transcendental equations. These estimators are easily evaluated with simple expressions.
\end{abstract}




\section{INTRODUCTION}

Recently, the $\alpha-\mu$ distribution has been become attractive for wireless communication fading modeling. The $\alpha-\mu$ distribution is most often referred to as the generalized gamma distribution [1] or the Stacy distribution [2] in the statistical literature although by now it is acknowledged that Amoroso's paper in Annali di Matematica [3] was probably the first work in which the generalized gamma distribution appeared. This distribution was proposed by Yacoub in [4], [5] to model the fading in nonlinear environments where the surfaces which cause diffuse scattering are spatially correlated. The main advantage of the $\alpha-\mu$ distribution is its mathematical simplicity and versatility even though includes important distributions as gamma, Nakagami-m, Weibull, one-side Gaussian and Rayleigh as particular cases. In [4], [5] the probability density function (PDF), cumulative distribution function (CDF) and moments of the $\alpha-\mu$ distribution were derived and related to the physical parameters of the fading assuming nonlinearities. The bivariate $\alpha-\mu$ distribution was studied in [6], where infinite series expressions were proposed for the joint PDF and CDF. In [7] a performance analysis of generalized-selection combining receivers over $\alpha$ - $\mu$ fading channels was carried out. Coulson et al. compared in [8] the $\alpha-\mu$ distribution with the Suzuki distribution to model the composite fast-fading and shadowing in narrowband wireless channels.

The $\alpha-\mu$ is a tri-parametric distribution. Many efforts have been carried out to estimate the parameters of the $\alpha-\mu$ distribution [4], [5], [9]-[13]. Since there are $\alpha-\mu$ distributions with rather different sets of $\alpha$ and $\mu$ parameters which can look very similar, the inference procedures to estimate $\alpha$ and $\mu$ parameters present significant problems. Maximum likelihood estimation (MLE) method is not straightforward. Two main approaches have been proposed: the maximization of the likelihood function [9] and the resolution of a scalar nonlinear equation derived from the likelihood equations [10]. However, the first method is not computationally efficient and the second can present persistent divergence. In [11] Huang and Hwan have found estimators of the parameters of the $\alpha-\mu$ distribution based on the moments method (MM), more efficient than MLE procedures for small number of samples. Even an iterative heuristic procedure by generating $\alpha-\mu$ random variables (RVs) has been proposed to estimate $\alpha$ and $\mu$ parameters in [12]. Recently, Song in [13] has obtained fast and globally convergent algorithms for estimating the three-parameters 
of the generalized gamma distribution based on scale-independent shape estimation (SISE) equations applied to real-time signals, video and image processing.

In this letter, a new approach for estimating the $\alpha-\mu$ distribution parameters based on the MM of the logarithmic $\alpha-\mu$ distribution is proposed. The advantages of the new proposed estimators are the following: i) the parameters of the $\alpha$ - $\mu$ distribution are calculated using direct equations from these new estimators and therefore their derivation is considerably simple; and ii) a unique solution exists for the parameters of this distribution with these novel estimators.

This letter is organized as follows: Section II presents the PDF, CDF and moments of the logarithmic $\alpha-\mu$ distribution and the fading margin which is a performance parameter used in wireless communications. In Section III, the estimators of the $\alpha-\mu$ distribution are derived calculating numerically the error due to the functions approximations. Section IV illustrates numerical examples, and finally the conclusions are discussed in Section V.

\section{THE LOGARITHMIC $\alpha-\mu$ DISTRIBUTION}

Let $r=\sqrt[\alpha]{\sum_{j=1}^{\mu}\left(x_{j}^{2}+y_{j}^{2}\right)}$ be the envelope of the sum of multipath components, where $x_{j}$ and $y_{j}$ are mutually independent Gaussian processes corresponding to the multipath component $j$-th, with $E\left(x_{j}\right)=E\left(y_{j}\right)=0$ and $E\left(x_{j}^{2}\right)=E\left(y_{j}^{2}\right)=\sigma^{2}$, where $E(\cdot)$ is the expectation operator. The PDF of $r$ is given by [4]

$$
p_{r}(r)=\frac{\alpha \mu^{\mu} r^{\alpha \mu-1}}{\Omega^{\alpha \mu} \Gamma(\mu)} \exp \left(-\mu \frac{r^{\alpha}}{\Omega^{\alpha}}\right), \quad r \geq 0,
$$

where $\Omega=\sqrt[\alpha]{2 \sigma^{2} \mu}, \Gamma(x)=\int_{0}^{\infty} t^{x-1} \exp (-t) d t$ is the gamma function $[14,(6.1 .1)]$, and

$$
\mu=\frac{E^{2}\left(r^{\alpha}\right)}{E\left(r^{2 \alpha}\right)-E^{2}\left(r^{\alpha}\right)}
$$

Note that in similar way to $[15,(120)], \mu$ can be extended to positive numbers, satisfying $\mu>0$. For $\mu=1$, the $\alpha-\mu$ distribution becomes the Weibull distribution. For $\alpha=1$ and $\alpha=2$ the $\alpha$ - $\mu$ distribution converts into the gamma and Nakagami- $m$ distributions, respectively. By setting $\alpha=2$ and $\mu=1$, we turn the $\alpha$ - $\mu$ distribution 
into the Rayleigh distribution. Also, the $\alpha-\mu$ distribution is equivalent to the onesided Gaussian distribution for $\alpha=2$ and $\mu=1 / 2$ [4].

The $k$-moment of the $\alpha-\mu$ distribution is given by [4], [5]

$$
E\left(r^{k}\right)=\frac{\Omega^{k} \Gamma\left(\mu+\frac{k}{\alpha}\right)}{\mu^{k / \alpha} \Gamma(\mu)} .
$$

We can define $\varepsilon=K \ln r$, where $K=20 / \ln (10)$. Using the elementary transformation of variables, the $\mathrm{PDF}$ of $\varepsilon$ is given by

$$
p_{\varepsilon}(\varepsilon)=\frac{1}{K} \frac{\alpha \mu^{\mu}}{\Omega^{\alpha \mu} \Gamma(\mu)} \exp \left(\frac{\alpha \mu}{K} \varepsilon-\frac{\mu}{\Omega^{\alpha}} e^{\frac{\alpha \varepsilon}{K}}\right), \quad-\infty<\varepsilon<\infty .
$$

The moment-generating function of $\varepsilon$ is calculated as

$$
\mathcal{M}_{\varepsilon}(s)=\int_{-\infty}^{\infty} p_{\varepsilon}(\varepsilon) e^{\varepsilon s} d \varepsilon=\int_{-\infty}^{\infty} \frac{1}{K} \frac{\alpha \mu^{\mu}}{\Omega^{\alpha \mu} \Gamma(\mu)} \exp \left(\left(\frac{\alpha \mu}{K}+s\right) \varepsilon-\frac{\mu}{\Omega^{\alpha}} e^{\frac{\alpha \varepsilon}{K}}\right) d \varepsilon .
$$

Using the transformation $u=\exp (\alpha \varepsilon / K)$ the integral of equation (5) can be expressed as

$$
\mathcal{M}_{\varepsilon}(s)=\frac{\mu^{\mu}}{\Omega^{\alpha \mu} \Gamma(\mu)} \int_{0}^{\infty} u^{\mu+\frac{K t}{\alpha}-1} \exp \left(-\frac{\mu}{\Omega^{\alpha}} u\right) d u,
$$

which is easily solved using [16, (3.381 4)] as

$$
\mathcal{M}_{\varepsilon}(s)=\left(\frac{\Omega^{\alpha}}{\mu}\right)^{\frac{K s}{\alpha}} \frac{\Gamma\left(\mu+\frac{K s}{\alpha}\right)}{\Gamma(\mu)} .
$$

From (7), we can derive the first three moments of $\varepsilon$ as

$$
\begin{aligned}
& m_{1} \triangleq E(\varepsilon)=\left(\frac{K}{\alpha}\right)\left(\psi(\mu)-\ln \left(\frac{\mu}{\Omega^{\alpha}}\right)\right) \\
& m_{2} \triangleq E\left(\varepsilon^{2}\right)=\left(\frac{K}{\alpha}\right)^{2}\left(\left(\psi(\mu)-\ln \left(\frac{\mu}{\Omega^{\alpha}}\right)\right)^{2}+\psi^{\prime}(\mu)\right), \\
& m_{3} \triangleq E\left(\varepsilon^{3}\right)=\left(\frac{K}{\alpha}\right)^{3}\left(\left(\psi(\mu)-\ln \left(\frac{\mu}{\Omega^{\alpha}}\right)\right)^{3}+3 \psi^{\prime}(\mu)\left(\psi(\mu)-\ln \left(\frac{\mu}{\Omega^{\alpha}}\right)\right)+\psi^{\prime \prime}(\mu)\right),
\end{aligned}
$$


where $\psi(x)=\frac{\partial \ln \Gamma(x)}{\partial x}$ is the psi (digamma) function $[14, \quad(6.3 .1)]$; and $\psi^{\prime}(x)=\frac{\partial \psi(x)}{\partial x}=\frac{\partial^{2} \ln \Gamma(x)}{\partial x^{2}}$ and $\psi^{\prime \prime}(x)=\frac{\partial^{2} \psi(x)}{\partial x^{2}}=\frac{\partial^{3} \ln \Gamma(x)}{\partial x^{3}}$ are the polygamma functions of first and second order, respectively $[14,(6.4 .1)]$.

The first central moments of $\varepsilon$ can be calculated from (8)-(10) as

$$
\begin{aligned}
& \mu_{2} \triangleq E\left[(\varepsilon-\bar{\varepsilon})^{2}\right]=\left(\frac{K}{\alpha}\right)^{2} \psi^{\prime}(\mu), \\
& \mu_{3} \triangleq E\left[(\varepsilon-\bar{\varepsilon})^{3}\right]=\left(\frac{K}{\alpha}\right)^{3} \psi^{\prime \prime}(\mu) .
\end{aligned}
$$

For $\alpha=2$, the $\alpha-\mu$ distribution becomes the Nakagami- $m$ distribution and (11), (12) are equivalent to $[15,(20)]$.

The CDF of $\varepsilon$ is derived by substituting $r=\exp (\varepsilon / K)$ in $[5,(8)]$ as

$$
F_{\varepsilon}(\varepsilon)=\frac{\gamma\left(\mu, \frac{\mu}{\Omega^{\alpha}} \exp \left(\frac{\alpha}{K} \varepsilon\right)\right)}{\Gamma(\mu)}, \quad-\infty<\varepsilon<\infty,
$$

where $\gamma(a, x)=\int_{0}^{x} t^{a-1} \exp (-t) d t$ is the lower incomplete gamma function $[14,(6.5 .2)]$. In wireless communications, it is useful to define the fading margin, denoted by $M F_{P}$, as the difference expressed in $\mathrm{dB}$ between the mean of the logarithmic distribution and the amplitude exceeded with a probability $P$. Then, for the $\alpha-\mu$ distribution, the fading margin is given by

$$
M F_{P}=m_{1}-F^{-1}(\alpha, \mu, \Omega, 1-P)
$$

where $F^{-1}(\alpha, \mu, \Omega, u)$ represents the inverse of the CDF of $\varepsilon$ given by (13), and $m_{1}$ is calculated in (8).

Therefore, the second term of $(14), F^{-1}(\alpha, \mu, \Omega, 1-P)$, can be expressed as

$$
F^{-1}(\alpha, \mu, \Omega, 1-P)=\frac{K}{\alpha} \ln \left(\frac{\Omega^{\alpha}}{\mu} Q^{-1}(\mu, P)\right)
$$


where $Q^{-1}(a, u)$ is the inverse of the regularized incomplete gamma function defined as $z=Q^{-1}(a, u) / u=\frac{\Gamma(a, z)}{\Gamma(a)}$ in $[17,(06.12 .02 .0001 .01)]$ and $\Gamma(a, z)=\Gamma(a)-\gamma(a, z)$ $[14,(6.4 .1)]$

From (8), (14) and (15), we can calculate the fading margin of the $\alpha$ - $\mu$ distribution as

$$
M F_{P}=\frac{K}{\alpha}\left(\psi(\mu)-\ln \left[Q^{-1}(\mu, P)\right]\right)
$$

Using the asymptotic expression of $Q^{-1}(a, x)$ when $x \rightarrow 1$ given by $[17$, $(06.12 .06 .0007 .01)]$

$$
Q^{-1}(a, x)=w+\frac{w^{2}}{a+1}+\frac{(3 a+5) w^{3}}{2(a+1)^{2}(a+2)}+\frac{(a(8 a+33)+31) w^{4}}{3(a+1)^{3}(a+2)(a+3)}+\ldots
$$

with $w=(-(x-1) \Gamma(a+1))^{1 / a}$, we can expand the fading margin for $P$ close to 1 as

$$
\begin{aligned}
& \underset{P \rightarrow 1}{M F_{P}}=\frac{K}{\alpha}\left(\psi(\mu)-\ln \left(((1-P) \Gamma(\mu+1))^{\frac{1}{\mu}}+\frac{((1-P) \Gamma(\mu+1))^{\frac{2}{\mu}}}{(\mu+1)}\right.\right. \\
& \left.+\frac{(3 \mu+5)((1-P) \Gamma(\mu+1))^{\frac{3}{\mu}}}{2(\mu+1)^{2}(\mu+2)}+\frac{(\mu(8 \mu+33)+31)((1-P) \Gamma(\mu+1))^{\frac{4}{\mu}}}{3(\mu+1)^{3}(\mu+2)(\mu+3)}+\ldots\right)
\end{aligned}
$$

\section{ESTIMATORS OF $\alpha-\mu$ DISTRIBUTION}

In this section, the estimators of the $\alpha-\mu$ distribution based on the MM are described. Later, we present new estimators using the logarithmic $\alpha-\mu$ distribution with the moments method.

We can define ${ }_{\beta} \mu[4,(23)]$ as

$$
{ }_{\beta} \mu=\frac{E^{2}\left(r^{\beta}\right)}{E\left(r^{2 \beta}\right)-E^{2}\left(r^{\beta}\right)}=\frac{\Gamma^{2}(\mu+\beta / \alpha)}{\Gamma(\mu+2 \beta / \alpha)-\Gamma^{2}(\mu+\beta / \alpha)} .
$$

Using (19), we can evaluate $\alpha$ and $\mu$ from ${ }_{\beta_{1}} \mu$ and ${ }_{\beta_{2}} \mu$ with numerical methods using the two following transcendental equations 


$$
\begin{gathered}
{ }_{\beta_{1}} \mu=\frac{\Gamma^{2}\left(\hat{\mu}+\beta_{1} / \hat{\alpha}\right)}{\Gamma\left(\hat{\mu}+2 \beta_{1} / \hat{\alpha}\right)-\Gamma^{2}\left(\hat{\mu}+\beta_{1} / \hat{\alpha}\right)}=\frac{\left(\frac{1}{N} \sum_{i=1}^{N} r_{i}^{\beta_{1}}\right)^{2}}{\left(\frac{1}{N} \sum_{i=1}^{N} r_{i}^{2 \beta_{1}}\right)-\left(\frac{1}{N} \sum_{i=1}^{N} r_{i}^{\beta_{1}}\right)^{2}}, \\
{ }_{\beta_{2}} \mu=\frac{\Gamma^{2}\left(\hat{\mu}+\beta_{2} / \hat{\alpha}\right)}{\Gamma\left(\hat{\mu}+2 \beta_{2} / \hat{\alpha}\right)-\Gamma^{2}\left(\hat{\mu}+\beta_{2} / \hat{\alpha}\right)}=\frac{\left(\frac{1}{N} \sum_{i=1}^{N} r_{i}^{\beta_{2}}\right)^{2}}{\left(\frac{1}{N} \sum_{i=1}^{N} r_{i}^{2 \beta_{2}}\right)-\left(\frac{1}{N} \sum_{i=1}^{N} r_{i}^{\beta_{2}}\right)^{2}},
\end{gathered}
$$

where $\hat{\alpha}$ and $\hat{\mu}$ are the estimators of the parameters $\alpha$ and $\mu$, respectively; and $r_{i}, i$ $=1, \ldots, N$ are the samples of the amplitudes, where $N$ is the number of samples.

Once $\hat{\alpha}$ has been calculated, the estimation of $\Omega$ parameter, denoted by $\hat{\Omega}$, can be easily obtained as

$$
\hat{\Omega}=\left(\frac{1}{N} \sum_{i=1}^{N} r_{i}^{\hat{\alpha}}\right)^{1 / \hat{\alpha}} .
$$

We define a new estimator, $\hat{\eta}$, as

$$
\hat{\eta}=\frac{\hat{\mu}_{2}^{3 / 2}}{\hat{\mu}_{3}}=\frac{\left[\frac{1}{N} \sum_{i=1}^{N}\left(K \ln r_{i}-\hat{m}_{1}\right)^{2}\right]^{3 / 2}}{\frac{1}{N} \sum_{i=1}^{N}\left(K \ln r_{i}-\hat{m}_{1}\right)^{3}},
$$

where

$$
\hat{m}_{1}=\frac{1}{N} \sum_{i=1}^{N} K \ln r_{i}
$$

and $\hat{\mu}_{2}$ and $\hat{\mu}_{3}$ are the estimators of the second and third central moments of the logarithmic $\alpha-\mu$ distribution, respectively. Note that $\hat{\eta}$ is an estimation of the multiplicative inverse of the logarithmic $\alpha-\mu$ distribution sample skewness [18].

Substituting (11) and (12) into (23), it yields

$$
\hat{\eta}=\frac{\left(\psi^{\prime}(\hat{\mu})\right)^{3 / 2}}{\psi^{\prime \prime}(\hat{\mu})} .
$$

The inverse of the function given by $(25)$, i.e. $\hat{\mu}=f(\hat{\eta})$, where $f(\cdot)$ represents a function, is evaluated numerically with a relative error less than $10^{-4}$. From this 
inverse function, the $\hat{\mu}$ estimator of the $\alpha-\mu$ distribution can be approximated using the least-squares method as

$$
\hat{\mu}=\left\{\begin{array}{cc}
\hat{\eta}^{2}+\frac{1}{2} & \hat{\eta} \leq-2.85 \\
-0.0773 \hat{\eta}^{4}-0.6046 \hat{\eta}^{3}-0.7949 \hat{\eta}^{2}-2.4675 \hat{\eta}-0.9208 & -2.85<\hat{\eta} \leq-0.6 . \\
-132.8995 \hat{\eta}^{3}-232.0659 \hat{\eta}^{2}-137.6303 \hat{\eta}-27.3616 & -0.6<\hat{\eta}<-0.5
\end{array}\right.
$$

Fig. 1 shows the $\hat{\mu}$ estimator approximation using (26) as a function of $\hat{\eta}$. Note that $\hat{\mu} \rightarrow 0$ for $\hat{\eta} \rightarrow-0.5$. The relative error of this approximation is less than $3.4 \cdot 10^{-3}$ using the approximation of the first interval (relative error of $3.4 \cdot 10^{-3}$ for $\hat{\eta}=-2.85$ ). The maximum relative error for the second interval approximation is $4.1 \cdot 10^{-2}$.

From (11), the estimation of the $\hat{\alpha}$ parameter can be easily obtained as

$$
\hat{\alpha}=K \sqrt{\frac{\psi^{\prime}(\hat{\mu})}{\hat{\mu}_{2}}},
$$

where $\hat{\mu}_{2}$ is the estimator of the second central moment of the logarithmic $\alpha-\mu$ distribution and $\hat{\mu}$ is the $\mu$ estimator of the $\alpha-\mu$ distribution, given by (26).

Since $\hat{\mu}$ as a function of $\hat{\eta}$ is a monotonically decreasing function, a unique solution exists for $\hat{\alpha}$ and $\hat{\mu}$ given a pair of $\hat{\eta}$ and $\hat{\mu}_{2}$ values.

\section{NUMERICAL RESULTS}

We have compared the performance of the two estimators using the Monte Carlo method: the central moment (CM) estimators proposed in [4], calculated solving (20) and (21) through numerical methods for $\beta_{1}=1, \beta_{2}=2$; and the skewness logarithmic (SL) estimators obtained from (23), (26) and (27).

In order to generate $\alpha-\mu$ distributed samples, it is useful to note that if $t$ is a gamma $\mathrm{RV}$, denoted by $t \sim \mathcal{G}\left(\mu, \Omega^{\alpha}\right)$, whose PDF is given by

$$
p_{t}(t)=\left(\frac{\mu}{\Omega^{\alpha}}\right)^{\mu} \frac{t^{\mu-1}}{\Gamma(\mu)} \exp \left(-\mu \frac{t}{\Omega^{\alpha}}\right), \quad t \geq 0
$$

then $r=\sqrt[\alpha]{t}$ is a $\alpha-\mu \mathrm{RV}[4]$ whose PDF is given by (1).

We have generated $N$ samples following an $\alpha-\mu$ distribution with the procedure described above. For convenience, we have set $\Omega=1$. 
We can calculate the mean of an estimator $\hat{s}$, denoted by $\overline{\hat{s}}_{M}$, as

$$
\overline{\hat{s}}_{M}=\frac{1}{M} \sum_{j=1}^{M} \hat{s}_{j}
$$

where $\hat{s}_{j}$ corresponds to each trial of the $\hat{s}$ estimator for a given distribution and $M$ is the number of trials of each RV. The sample confidence region of the $\hat{s}$ estimator, denoted by $C_{\hat{s}_{M}}$, can be defined as

$$
C_{s_{M}}= \pm 2 \sigma_{s_{M}}= \pm 2 \sqrt{\left(\frac{1}{M} \sum_{j=1}^{M} \hat{s}_{j}^{2}-\overline{\hat{s}}_{M}^{2}\right)}= \pm 2 \sqrt{\left(\frac{1}{M} \sum_{j=1}^{M} \hat{s}_{j}^{2}-\left(\frac{1}{M} \sum_{j=1}^{M} \hat{s}_{j}\right)^{2}\right)}
$$

where $\sigma_{\hat{s}_{M}}$ is the sample standard deviation of the $\hat{s}$ estimator. The sample confidence region is useful for examining the variations of an estimator in terms of the others estimators and the number of samples $N$ [19]. $M=500$ trials of each $\alpha-\mu$ $\mathrm{RV}$ have been carried out to obtain statistical parameters of each estimator.

Fig. 2 shows the sample mean and the sample confidence region of $\hat{\mu}$ for $N=10000$ samples and $\alpha=1.2$ using the CM and SL estimators. Since the sample means of $\hat{\mu}$ are slightly above the reference line with a slope equal to 1 , both estimators are very slightly positive biased. The sample confidence region is slightly broader in the CM than in the SL estimator for $\alpha=1.2$.

The normalized mean square error (NMSE) of a generic estimator $\hat{s}$ is defined as

$$
\mathrm{NMSE}=\frac{\frac{1}{M} \sum_{j=1}^{M}\left(\hat{s}_{j}-s\right)^{2}}{s^{2}},
$$

where $s$ is the parameter of the distribution to be estimated.

Fig. 3 shows a comparison of NMSE of $\hat{\mu}$ for the estimators CM and SL as a function of $\mu$ for $\alpha=0.8,1.2,1.8$ and $N=10000$ samples. The NMSE is considerably higher in the CM compared to the SL estimator with $\alpha=0.8$. For $\alpha=$ 1.2, the NMSE in the SL is also lower than in the CM. Otherwise, the NMSE is similar in the CM and SL estimators with $\alpha=1.8$. From these curves, it can be shown that the NMSE of $\hat{\mu}$ for the SL estimator is independent on the value of $\mu$, increasing with $\mu$ from 1.5 to 10 .

In Fig. 4 the NMSE of the $\hat{\mu}$ estimator is shown in terms of the number of samples for the CM and SL estimator methods. We have plotted the NMSE of the $\hat{\mu}$ 
estimator from $N=10000$ to 100000 samples for three pairs of $\alpha$ and $\mu$ parameters: $\alpha=1.1, \mu=1.3 ; \alpha=1.6, \mu=1.3$; and $\alpha=2.2, \mu=1.8$. The NMSE of $\hat{\mu}$ using the $\mathrm{CM}$ estimator is strongly dependent on $\alpha$. For low values of $\alpha$ (e.g. $\alpha=1.1$ ), the NMSE of $\hat{\mu}$ with the CM estimator method increases significantly. Otherwise, the NMSE of $\hat{\mu}$ scarcely depends on $\alpha$ using the SL estimator for the values of these simulations. The NMSE of $\hat{\mu}$ presents a poorer behaviour in the CM than in the SL estimator for high values of $N$ (for $N>40000$; and $\alpha=1.6, \mu=1.3$ or $\alpha=2.2, \mu=$ 1.8). Obviously, since SL estimators do not require numerical methods to be solved, the computer simulation time is considerably higher using the $\mathrm{CM}$ estimator, increased significantly as $N$.

Finally, Fig. 5 shows the NMSE of the $\hat{\mu}$ estimator as a function of the number of samples for the SL and the SISE estimator methods. This NMSE has been plotted from $N=10000$ to 100000 samples for three pairs of $\alpha$ and $\mu$ parameters: $\alpha=0.8, \mu$ $=4.3 ; \alpha=3.9, \mu=0.7$; and $\alpha=2.5, \mu=1.9$. The SISE estimators have been calculated using $[13,(12)]$ with the Newston-Raphson algorithm. From Fig. 5, it can be observed that the NMSE of $\hat{\mu}$ using the SISE estimator depends strongly on $\alpha$. For a given value of $N$ dependent on $\alpha$ (e.g. $N<3000$ for $\alpha=0.8$ and $\mu=4.3 ; N<$ 20000 for $\alpha=2.5$ and $\mu=1.9$ ), the SL estimator provides a NMSE less than using the SISE estimator method. Nevertheless, the NMSE of $\hat{\mu}$ using the SISE estimator decreases substantially for low values of $\mu$ (e.g. $\mu=0.7$ ). Moreover, the NMSE of $\hat{\mu}$ as a function of $N$ in a logarithmic plot using the SL estimator can be approximated by a straight line whose slope is approximately constant.

\section{CONCLUSION}

New estimators of the $\alpha-\mu$ distribution based on the moments method have been obtained using the logarithm of a $\alpha-\mu \mathrm{RV}$ avoiding the numerical resolution of the central moments transcendental equations of the $\alpha-\mu$ distribution. These estimators are calculated from the multiplicative inverse of the logarithmic $\alpha-\mu$ distribution skewness. They can be of interest for estimating the envelope of the received signal in nonlinear propagation mediums. The NMSE of $\hat{\mu}$ with the skewness logarithmic estimators provides superior behaviour to solve central moments equations for low $\alpha$ values (less than 1.2). Moreover, the NMSE of $\hat{\mu}$ with these proposed skewness logarithmic estimators is smaller than using both the central moment and the scale 
independent shape estimation methods for large number of samples and values of $\mu$ higher than 1.2.

\section{REFERENCES}

[1] C. Kleiber and S. Kotz, Statistical Size Distributions in Economics and Actuarial Sciences. Hoboken, NY: Wiley, 2003.

[2] E. W. Stacy, "A generalization of the gamma distribution," Ann. Math. Stat., vol. 33, pp. 1187-1192, Sep. 1962.

[3] L. Amoroso, "Richerche intorno alla curva dei redditi," Annali di Matematica Pura ed Applicata, vol. 2, pp. 123-159, Dec. 1925.

[4] M. D. Yacoub, "The $\alpha-\mu$ distribution: a general fading distribution," in IEEE Personal, Indoor and Mobile Radio Communications, PIMRC-02, vol. 2, pp. 629-633, Sep. 2002.

[5] - "The $\alpha-\mu$ distribution: A physical fading model for the Stacy distribution," IEEE Trans. Veh. Technol., vol. 56, pp. 27-34, Jan. 2007.

[6] T. Piboongungon, V. A. Aalo, C. D. Iskander and G. P. Efthymoglou, "Bivariate generalized gamma distribution with arbitrary fading parameters," Electron. Lett., vol. 41, pp. 709-710, Jun. 2005.

[7] P. S. Bithas, N. C. Sagias, and P. T. Mathiopoulos, "GSC diversity receivers over generalized-gamma fading channels," IEEE Commun. Lett., vol. 11, pp. 964-966, Dec. 2007.

[8] A. J. Coulson, A. G. Williamson, and R. G. Vaughan, "Improved fading distribution for mobile radio," IEE Proc. Commun., vol. 145, pp. 197-202, Jun. 1998.

[9] J. F. Lawless, "Inference in the generalized gamma and log gamma distributions," Technometrics, vol. 22, 409-419, 1980.

[10] H. W. Hager and L. J. Bain, "Inferential procedures for the generalized gamma distribution," Journal of the American Statistical Association, vol. 65, 334-342, 1970.

[11] P. -H. Huang and T. -Y. Hwang, "On new moment estimation of parameters of the generalized gamma distribution using its characterization," Taiwanese Journal of Mathematics, vol. 10, pp. 1083-1093, Jun. 2006.

[12] O. Gomes, C. Combes, and A. Dussauchoy, "Parameter estimation of the generalized gamma distribution," Mathematics and Computers in Simulation, Elsevier, vol. 79, pp. 955-963, 2008. 
[13] K.-S. Song, "Globally convergent algorithms for estimating generalized gamma distributions in fast signal and image processing," IEEE Trans. Image Process., vol. 17, pp. 1233-1250, Aug. 2008.

[14] M. Abramowitz and I. Stegun. Handbook of Mathematical Functions. Washington, DC: National Bureau of Standards, 10 ${ }^{\text {th }}$ Ed., 1972.

[15] M. Nakagami, "The $m$-distribution-A general formula of intensity distribution of rapid fading," in Statistical Methods of Radio Wave Propagation, W. G. Hoffman Ed., Pergamon Press, Oxford, UK, 1960.

[16] I. S. Gradsthteyn and I. M. Ryzhik. Table of Integrals, Series and Products. San Diego, CA: Academic, $7^{\text {th }}$ ed., 2007.

[17] WolframMathworld. (2009, Oct). [Online]. Available: http://functions.wolfram.com/ GammaBetaErf/InverseGammaRegularized/ (Last access 10/20/2009).

[18] E. W. Stacy and G. A. Mihram, "Parameter estimation for a generalized gamma distribution," Technometrics, vol. 7, pp. 349-358, Aug. 1965.

[19] A. Abdi and M. Kaveh, "Performance comparison of three different estimators for the Nakagami m parameter," IEEE Commun. Lett., vol. 4, pp. 119-121, Apr. 2000 


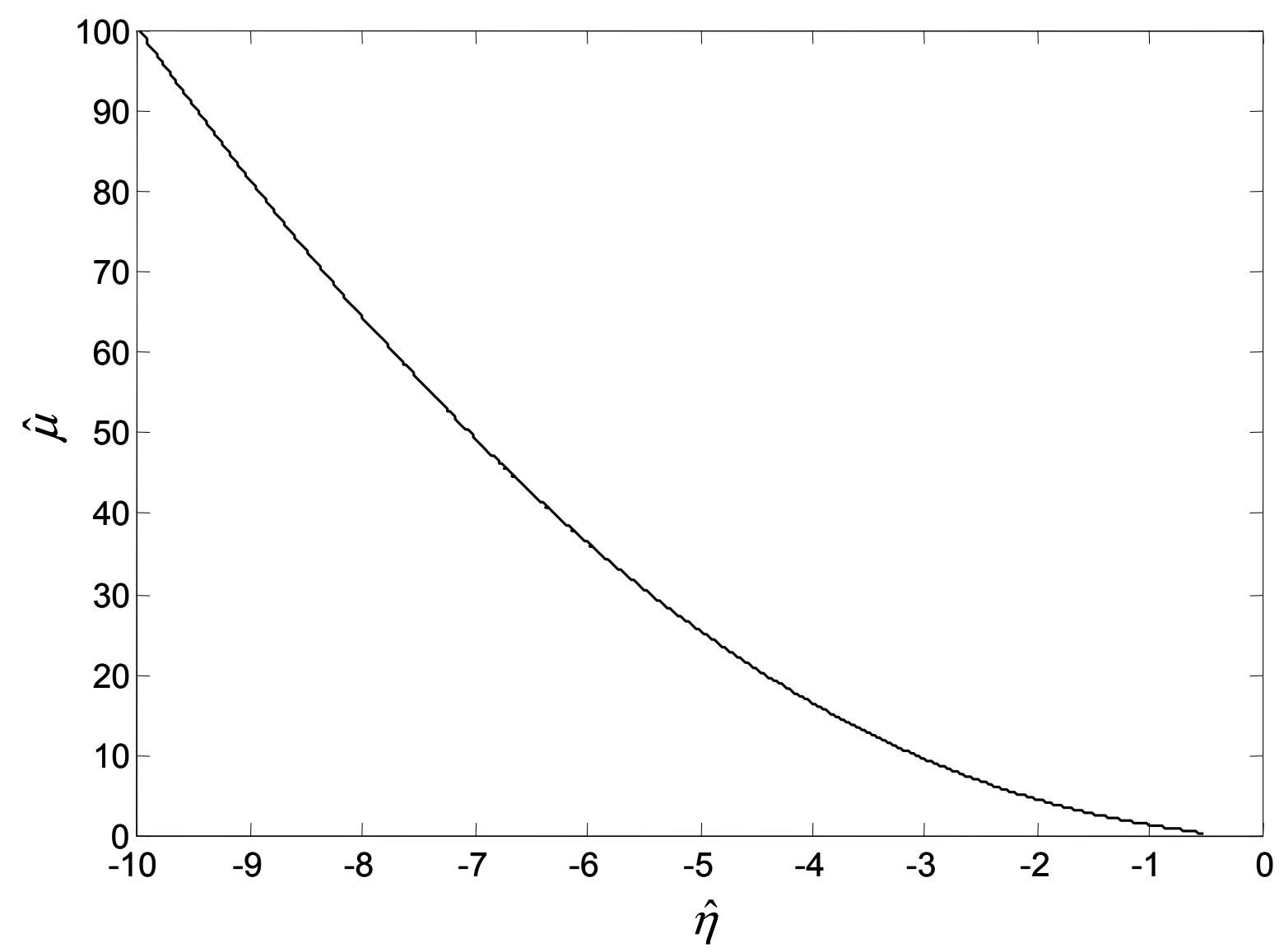

Figure 1. Polynomial approximation of $\hat{\mu}$ as a function of the $\hat{\eta}$ estimator. 

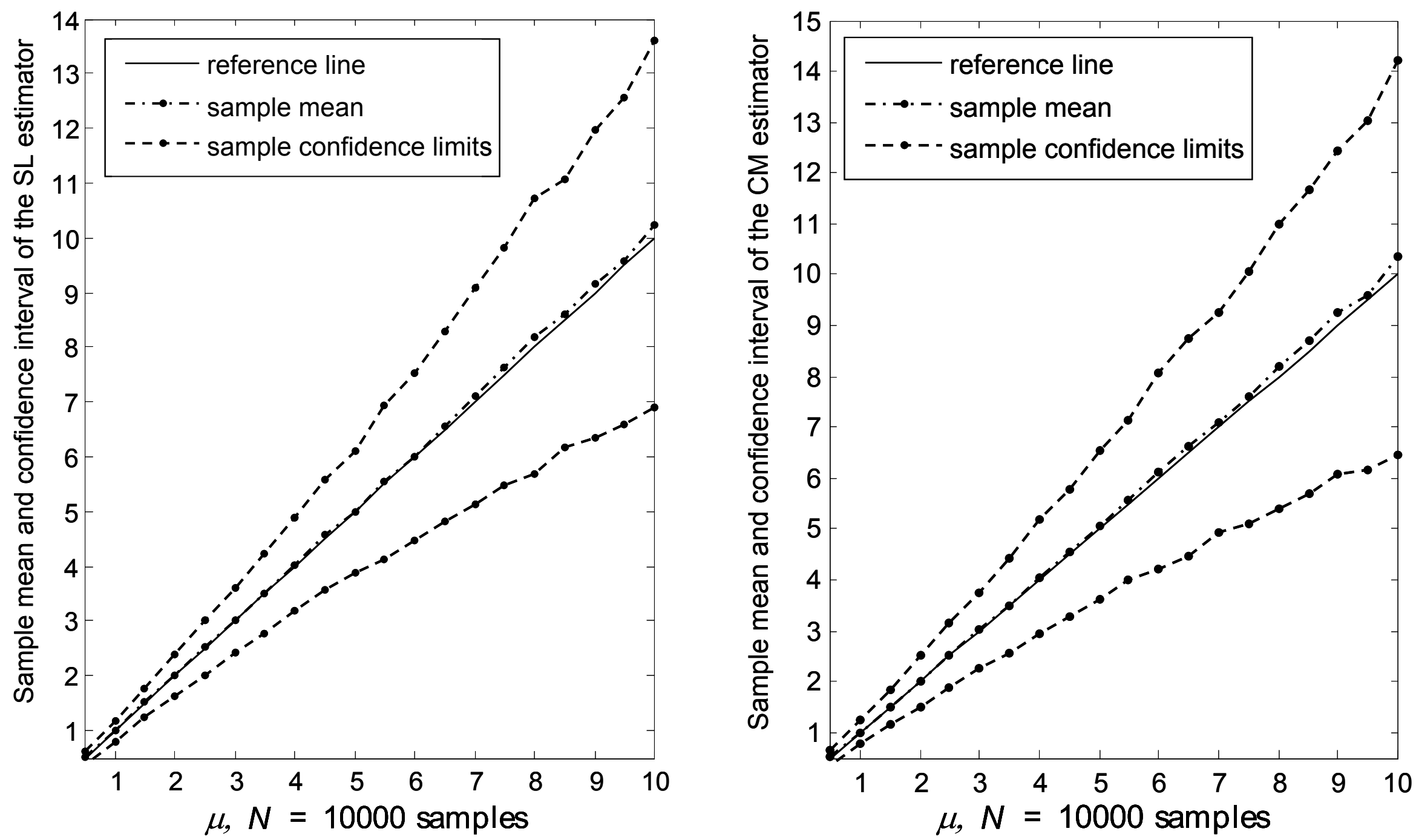

Figure 2. Sample mean and the sample confidence region of $\hat{\mu}$ for the skewness logarithmic (SL) and central moment (CM) estimators $(N=10000, \alpha=1.2)$. 


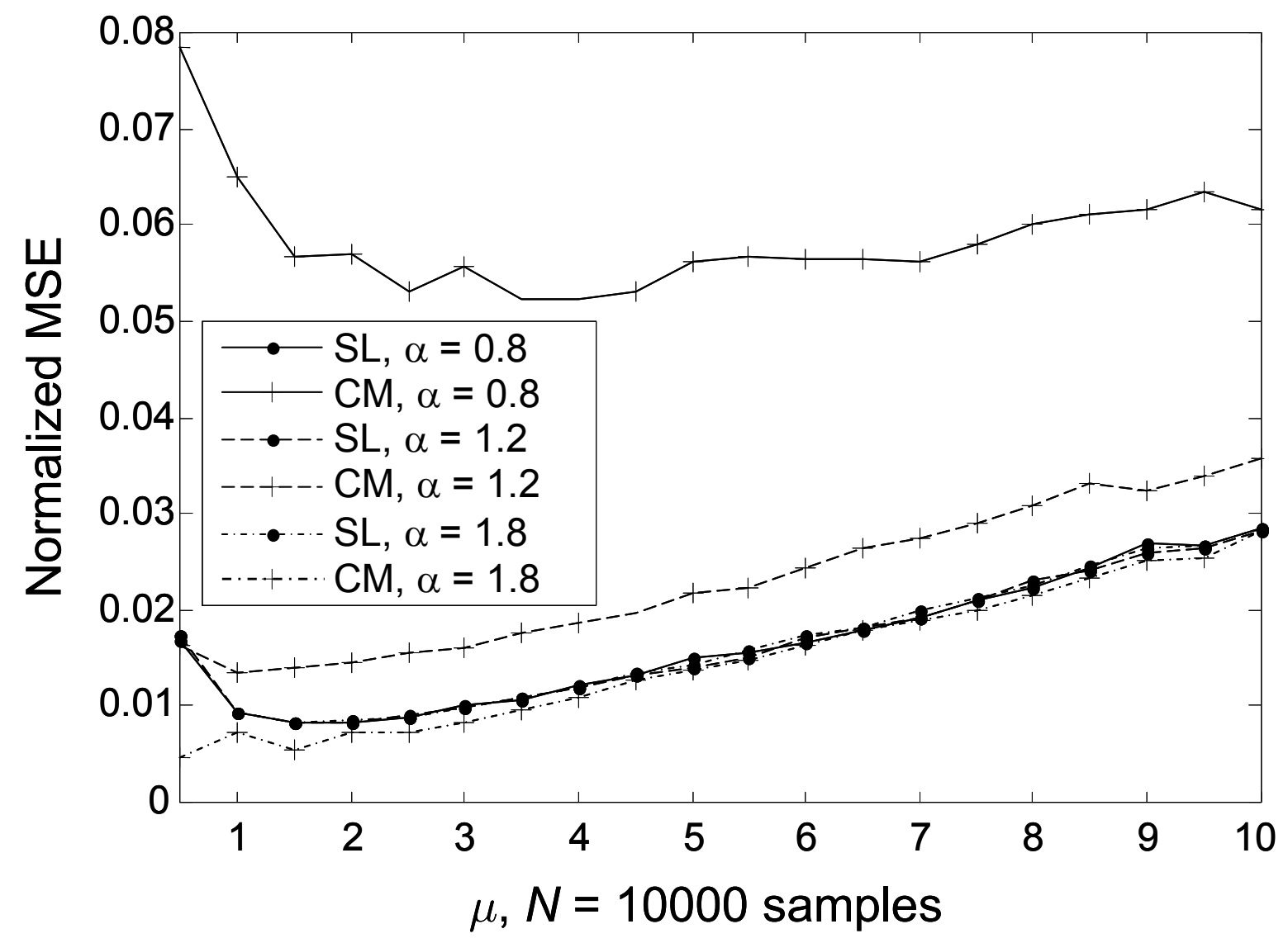

Figure 3. Normalized mean square error (MSE) of $\hat{\mu}$ estimator for the central moment $(\mathrm{CM})$ and the skewness logarithmic (SL) methods as a function of $\mu(N=$ 10000 samples and $\alpha=0.8,1.2$ and 1.8$)$. 


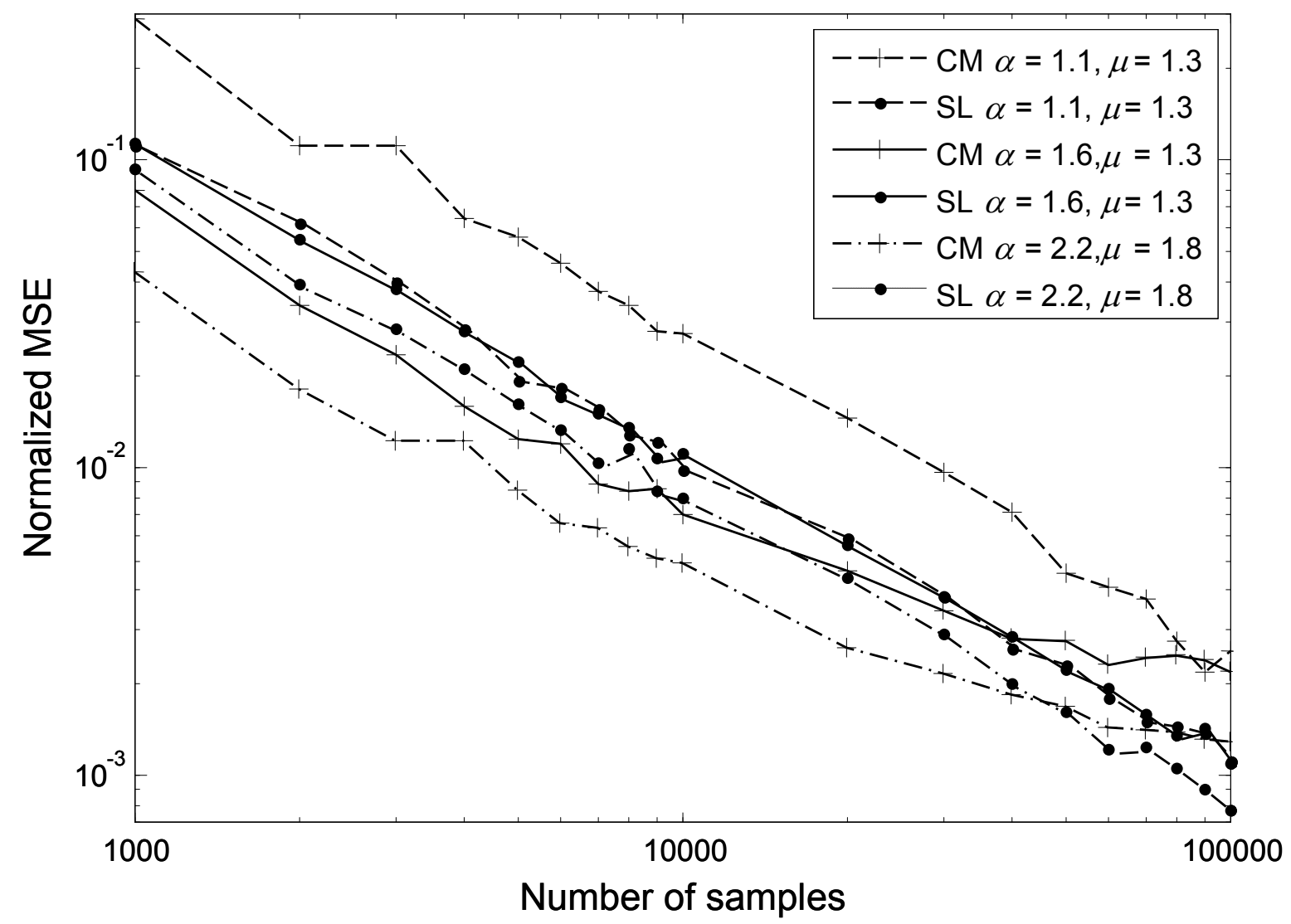

Figure 4. Normalized mean square error (MSE) of $\hat{\mu}$ estimator for the central moment $(\mathrm{CM})$ and the skewness logarithmic (SL) methods as a function of the number of samples. Parameters used in simulations: $\alpha=1.1, \mu=1.3 ; \alpha=1.6, \mu=$ $1.3 ;$ and $\alpha=2.2, \mu=1.8$. 


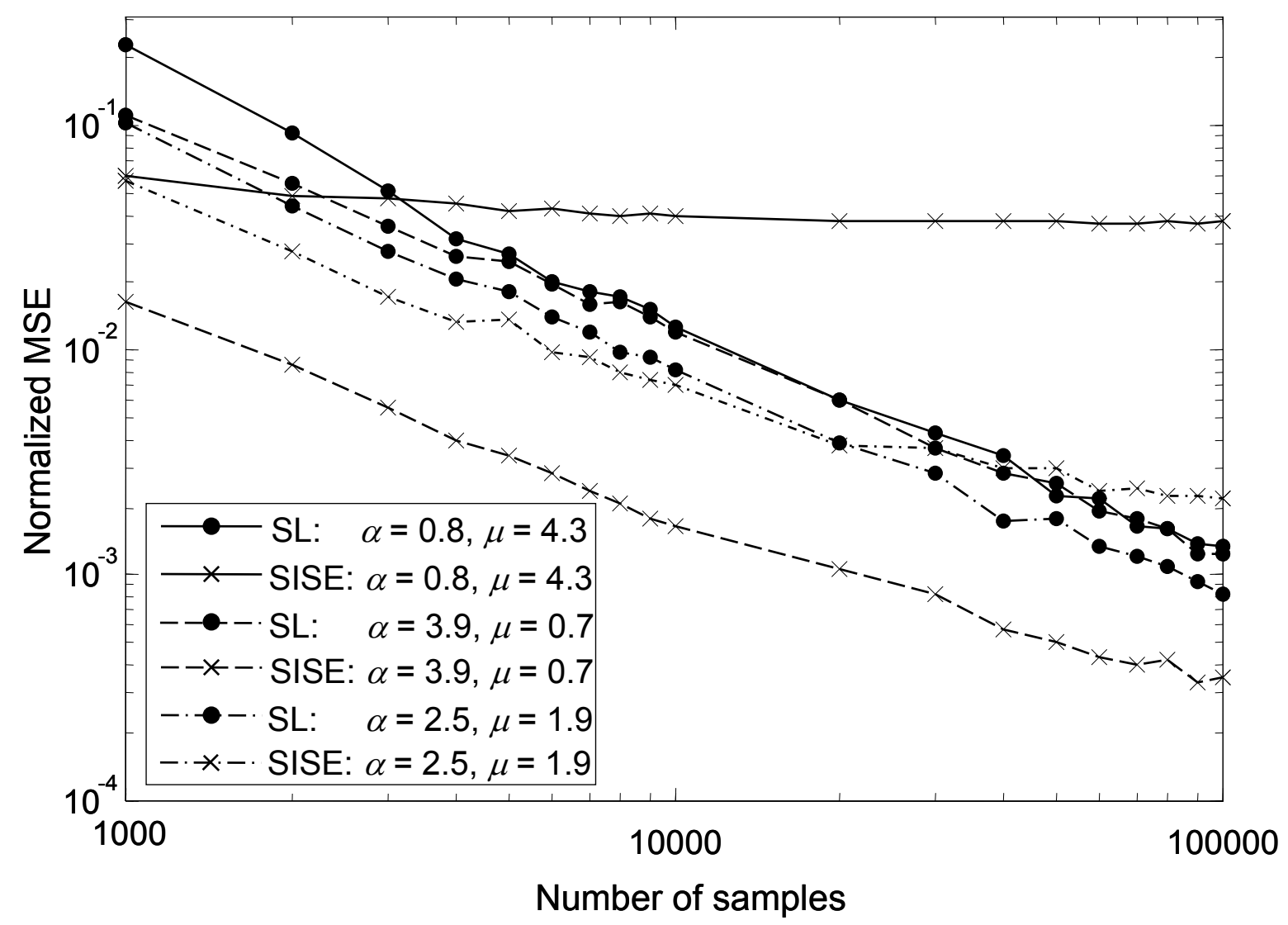

Figure 5. Normalized mean square error (MSE) of $\hat{\mu}$ estimator for the scaleindependent shape estimator (SISE) and the skewness logarithmic (SL) methods as a function of the number of samples. Parameters used in simulations: $\alpha=0.8, \mu=4.3$; $\alpha=3.9, \mu=0.7$; and $\alpha=2.5, \mu=1.9$. 